\title{
CONSTRUCTION OF THE GENERALISED $q$-DERIVATIVE OPERATORS
}

\author{
Dayanand Parashar \\ Department of Physics, A.R.S.D. College, University of Delhi \\ New Delhi - 110021, India \\ Deepak Parashar \\ Department of Mathematics, University of Wales Swansea, Singleton Park \\ Swansea SA2 8PP, United Kingdom \\ D.Parashar@swansea.ac.uk \\ http://www-maths.swan.ac.uk/staff/dp/
}

\begin{abstract}
This investigation pertains to the construction of a class of generalised deformed derivative operators which furnish the familiar finite difference and the $q$-derivatives as special cases. The procedure involves the introduction of a linear operator which is multiplicative over functions of a real variable. The validity of the general prescriptions is ascertained by considering suitable examples of such derivatives and constructing their eigenfunctions explicitly. The relationship of a particular version of the operator with the one-dimensional Möbius transformation is also established.
\end{abstract}

MSC: 05A30; 26A24; 81R50

Keywords: $q$-derivatives; $q$-calculus; Möbius transformations

J. Geom. Phys. 48 (2003) 297-308 


\section{Introduction}

Ever since the pioneering work of Jackson [1, there have been numerous attempts [2, 3, 4] to carry out investigation into various aspects of deformations which have played a crucial role in the understanding of important mathematical and physical concepts. A concrete realisation of such attributes is easily traced to the emergence of quantum groups [5, 6, embodying appropriate deformations of algebraic and geometric structures in a particularly elegant formulation. While there are several ways in which to study deformations, perhaps the most essential ingredients in such a study would invariably comprise, for instance, the $q$-deformed derivative operators, coordinate multiplication operators, etc., among others. Significantly, these $q$-deformed operators have met with tremendous success when applied to a host of problems, most notably in the realm of oscillator algebra 2, 7, 8, and $q$-calculus [9. This offers the main motivation to seek a generalisation of these operators in order to be able to try applications of the underlying prescriptions to a wider class of systems.

In Sec. 2, we construct a generalised deformed derivative operator and study the corresponding eigenfunctions. Sec. 3 is devoted to a discussion of specific examples of the generalised version of this operator where we explicitly demonstrate the salient features for the purpose of applications. A possible connection of the deformed operator with the reduced Möbius transformation operator is discussed in Sec. 4. Concluding remarks form contents of Sec. 5 .

\section{The Deformed Derivative}

To facilitate construction of the generalised deformed derivative, we consider a linear operator $\hat{\Omega}$ whose action on the product of two functions, say $f(x)$ and $g(x), x \in \mathbb{R}$, can be written as

$$
\hat{\Omega}\{f(x) g(x)\}=\{\hat{\Omega} f(x)\}\{\hat{\Omega} g(x)\}=f(\hat{\Omega} x) g(\hat{\Omega} x)
$$

clearly displaying the property that $\hat{\Omega}$ is multiplicative over functions of $x$. We further assign to $\hat{\Omega}$ dependence on a parameter $\lambda$ such that $\hat{\Omega} \rightarrow I$ (the identity operator) in the limit $\lambda \rightarrow$ 0 . This requirement is necessitated by the desire to accomplish meaningful comparisons with the more familiar derivative operators. In particular, the translation operator $\Omega=\hat{H}=e^{h \partial_{x}}$ such that $\hat{H} f(x)=f(x+h)$, while the $q$-dilation operator $\Omega=\hat{Q}=e^{\ln (q) x \partial_{x}}$ such that $\hat{Q} f(x)=f(q x)$ form examples of such an operator. An appropriate generalisation of the deformed derivative operator is constructed through the definition

$$
{ }_{\Omega} D_{x}=\left\{\frac{1}{(\hat{\Omega}-I) x}\right\}(\hat{\Omega}-I)
$$

which, operating on any function $f(x)$, gives

$$
{ }_{\Omega} D_{x} f(x)=\frac{f(\hat{\Omega} x)-f(x)}{(\hat{\Omega} x)-x}
$$

It follows immediately that ${ }_{\Omega} D_{x} x=1$ and ${ }_{\Omega} D_{x} c=0$ for any constant $c$.

Proposition 1 The deformed derivative ${ }_{\Omega} D_{x}$ obeys two modified Leibniz rules

$$
\begin{aligned}
& { }_{\Omega} D_{x}\{f(x) g(x)\}=\left\{{ }_{\Omega} D_{x} f(x)\right\} g(x)+\{\hat{\Omega} f(x)\}{ }_{\Omega} D_{x} g(x) \\
& { }_{\Omega} D_{x}\{f(x) g(x)\}=f(x)\left\{{ }_{\Omega} D_{x} g(x)\right\}+\left\{{ }_{\Omega} D_{x} f(x)\right\} \hat{\Omega} g(x)
\end{aligned}
$$

and the chain rule

$$
{ }_{\Omega} D_{x} f(g(x))={ }_{\Omega} D_{g(x)} f(g(x)){ }_{\Omega} D_{x} g(x)
$$




\section{Proof:}

$$
\begin{aligned}
{ }_{\Omega} D_{x}\{f(x) g(x)\}= & \frac{1}{(\hat{\Omega}-I) x}\{\hat{\Omega} f(x) g(x)-f(x) g(x)\} \\
= & \frac{1}{(\hat{\Omega}-I) x}\{[f(\hat{\Omega} x)] g(x)-[f(\hat{\Omega} x)] g(x) \\
& +f(\hat{\Omega} x) g(\hat{\Omega} x)-f(x) g(x)\} \\
= & \frac{1}{(\hat{\Omega}-I) x}\{[f(\hat{\Omega} x)-f(x)] g(x)+f(\hat{\Omega} x)[g(\hat{\Omega} x)-g(x)]\} \\
= & \left\{\frac{f(\hat{\Omega} x)-f(x)}{(\hat{\Omega} x)-x}\right\} g(x)+f(\hat{\Omega} x)\left\{\frac{g(\hat{\Omega} x)-g(x)}{(\hat{\Omega} x)-x}\right\} \\
= & \left\{{ }_{\Omega} D_{x} f(x)\right\} g(x)+\hat{\Omega} f(x)\left\{{ }_{\Omega} D_{x} g(x)\right\}
\end{aligned}
$$

The second Leibniz rule (2.5) follows from the first if we simply interchange the roles of $f$ and $g$. To prove the modified chain rule, we have

$$
\begin{aligned}
{ }_{\Omega} D_{x}\{f \circ g\}(x) & =\frac{\{f \circ g\}(\hat{\Omega} x)-\{f \circ g\}(x)}{(\hat{\Omega} x)-x} \\
& =\left\{\frac{f(g(\hat{\Omega} x))-f(g(x))}{g(\hat{\Omega} x)-g(x)}\right\}\left\{\frac{g(\hat{\Omega} x)-g(x)}{(\hat{\Omega} x)-x}\right\} \\
& =\left\{\frac{(\hat{\Omega}-I) f(g(x))}{(\hat{\Omega}-I) g(x)}\right\}\left\{\frac{(\hat{\Omega}-I) g(x)}{(\hat{\Omega}-I) x}\right\} \\
& ={ }_{\Omega} D_{g(x)} f(g(x))_{\Omega} D_{x} g(x)
\end{aligned}
$$

In the limit $\lambda \rightarrow 0$, we see that ${ }_{\Omega} D_{x} \rightarrow \frac{d}{d x}=\partial_{x}$. Note that this generalised derivative specialises to the familiar finite difference derivative ${ }_{h} \Delta_{x}$ :

$$
{ }_{h} \Delta_{x} f(x)=\frac{f(x+h)-f(x)}{h}
$$

and the $q$-derivative ${ }_{q} D_{x}$ :

$$
{ }_{q} D_{x} f(x)=\frac{f(q x)-f(x)}{(q-1) x}
$$

as expected.

\subsection{Inverse deformed derivative $\left({ }_{\Omega} D_{x}^{-1}\right)$}

The nonsingular behaviour of the operator ${ }_{\Omega} D_{x}$ can be ascertained only if the existence of its inverse ${ }_{\Omega} D_{x}^{-1}$ is guaranteed. Consequently, the condition ${ }_{\Omega} D_{x} \circ{ }_{\Omega} D_{x}^{-1}=I$ must necessarily be satisfied on analytic functions. We, therefore, proceed to define such an inverse. The operation of the deformed derivative ${ }_{\Omega} D_{x}$ on an arbitrary analytic function $f(x)$ can be rewritten in the form

$$
{ }_{\Omega} D_{x} f(x)=\frac{1}{(I-\hat{\Omega}) x}(I-\hat{\Omega}) f(x)
$$

which allows us to formally invert the operator ${ }_{\Omega} D_{x}$ to get the inverse operator ${ }_{\Omega} D_{x}^{-1}$ through the definition

$$
{ }_{\Omega} D_{x}^{-1}=\sum_{j=0}^{\infty} \hat{\Omega}^{j}\{(I-\hat{\Omega}) x\}
$$


As an illustrative example, let us compute the operation of ${ }_{\Omega} D_{x}^{-1}$ on $f(x)=x^{n}$ (say). We then have

$$
\begin{aligned}
{ }_{\Omega} D_{x}^{-1} x^{n} & =\sum_{j=0}^{\infty} \hat{\Omega}^{j}\{(I-\hat{\Omega}) x\} x^{n} \\
& =\sum_{j=0}^{\infty} \hat{\Omega}^{j}\left\{x^{n+1}-(\hat{\Omega} x) x^{n}\right\} \\
& =\sum_{j=0}^{\infty}\left\{\hat{\Omega}^{j} x^{n+1}-\left(\hat{\Omega}^{j+1} x\right)\left(\hat{\Omega}^{j} x^{n}\right)\right\}
\end{aligned}
$$

The operation of ${ }_{\Omega} D_{x}$ on (2.9) gives

$$
\begin{aligned}
{ }_{\Omega} D_{x} \circ{ }_{\Omega} D_{x}^{-1} x^{n}= & { }_{\Omega} D_{x} \sum_{j=0}^{\infty}\left\{\hat{\Omega}^{j} x^{n+1}-\left(\hat{\Omega}^{j+1} x\right)\left(\hat{\Omega}^{j} x^{n}\right)\right\} \\
= & \left(\frac{1}{(\hat{\Omega} x)-x}\right)\left\{\hat{\Omega} \sum_{j=0}^{\infty}\left\{\hat{\Omega}^{j} x^{n+1}-\left(\hat{\Omega}^{j+1} x\right)\left(\hat{\Omega}^{j} x^{n}\right)\right\}\right. \\
& \left.-\sum_{j=0}^{\infty}\left\{\hat{\Omega}^{j} x^{n+1}-\left(\hat{\Omega}^{j+1} x\right)\left(\hat{\Omega}^{j} x^{n}\right)\right\}\right\} \\
= & \left(\frac{1}{(\hat{\Omega} x)-x}\right)\left\{\sum _ { j = 0 } ^ { \infty } \left\{\hat{\Omega}^{j+1} x^{n+1}-\left(\hat{\Omega}^{j+2} x\right)\left(\hat{\Omega}^{j+1} x^{n}\right)\right.\right. \\
& \left.-\left(\frac{1}{(\hat{\Omega} x)-x}\right)\left\{\hat{\Omega} x^{j} x^{n+1}+\left(\hat{\Omega}^{j+1} x\right)\left(\hat{\Omega}^{j} x^{n}\right)\right\}\right\} \\
& \\
= & \left(\frac{1}{(\hat{\Omega} x)-x}\right)\left\{(\hat{\Omega} x) x^{2} x^{n+1}-\left(\hat{\Omega}^{3} x\right)\left(\hat{\Omega}^{2} x^{n}\right)-\left(\hat{\Omega} x^{n+1}\right)+\left(\hat{\Omega}^{2} x\right)\left(\hat{\Omega} x^{n}\right)+\cdots\right\} \\
= & x^{n}
\end{aligned}
$$

Hence the necessary condition

$$
{ }_{\Omega} D_{x} \circ{ }_{\Omega} D_{x}^{-1}=I
$$

is satisfied by the inverse derivative ${ }_{\Omega} D_{x}^{-1}$.

\subsection{Eigenfunctions}

The eigenfunctions of the deformed derivative operator ${ }_{\Omega} D_{x}$ can be shown to admit a product representation. The following proposition justifies this statement.

Proposition 2 If

$$
{ }_{\Omega} D_{x} E_{\Omega}(x)=E_{\Omega}(x)
$$

then the function $E_{\Omega}(x)$ is given by

$$
E_{\Omega}(x)=\prod_{j=0}^{\infty} \frac{1}{1+\hat{\Omega}^{j}(\hat{\Omega}-I) x}
$$


Proof: Let the function $E_{\Omega}(x)$ be defined as

$$
E_{\Omega}(x)=\prod_{j=0}^{\infty} c_{j}(x)
$$

where $c_{j}(x)$ is a sequence of functions of $x$

$$
c_{j}(x)=\hat{\Omega}^{j} c_{0}(x)=c_{0}\left(\hat{\Omega}^{j} x\right)
$$

Then

$$
\begin{aligned}
{ }_{\Omega} D_{x} E_{\Omega}(x) & =\left\{\frac{1}{(\hat{\Omega}-I) x}\right\}(\hat{\Omega}-I) \prod_{j=0}^{\infty} c_{j}(x) \\
& =\left\{\frac{1}{(\hat{\Omega}-I) x}\right\}\left\{\prod_{j=0}^{\infty} \hat{\Omega} c_{j}(x)-\prod_{j=0}^{\infty} c_{j}(x)\right\} \\
& =\left\{\frac{1}{(\hat{\Omega}-I) x}\right\}\left\{\prod_{j=0}^{\infty} c_{j+1}(x)-\prod_{j=0}^{\infty} c_{j}(x)\right\} \\
& =\left\{\frac{1}{(\hat{\Omega}-I) x}\right\}\left\{\prod_{j=0}^{\infty} \frac{c_{j}(x)}{c_{0}(x)}-\prod_{j=0}^{\infty} c_{j}(x)\right\} \\
& =\left\{\frac{1}{(\hat{\Omega}-I) x}\right\}\left\{c_{0}^{-1}(x)-1\right\} \prod_{j=0}^{\infty} c_{j}(x) \\
& =\left\{\frac{1}{(\hat{\Omega}-I) x}\right\}\left\{c_{0}^{-1}(x)-1\right\} E_{\Omega}(x)
\end{aligned}
$$

Since the function $c_{0}(x)$ (cf. (2.14) ) is an arbitrary function of $x$, we may choose

$$
c_{0}^{-1}(x)=1+(\hat{\Omega} x)-x
$$

so that the functions $c_{j}(x)$ can be cast in the form

$$
\begin{aligned}
c_{j}(x) & =\hat{\Omega}^{j} c_{0}(x) \\
& =c_{0}\left(\hat{\Omega}^{j} x\right) \\
& =\frac{1}{1+\hat{\Omega}^{j}(\hat{\Omega}-I) x}
\end{aligned}
$$

which, in turn, gives the desired eigenfunction

$$
E_{\Omega}(x)=\prod_{j=0}^{\infty} \frac{1}{1+\hat{\Omega}^{j}(\hat{\Omega}-I) x}
$$

In view of the relation ${ }_{\Omega} D_{x} E_{\Omega}(x)=E_{\Omega}(x)$, the eigenfunction $E_{\Omega}(x)$ can be interpreted as the $\Omega$-exponential function. An alternative description of the eigenfunctions $E_{\Omega}(x)$ proceeds via the series representation in terms of the inverse operator ${ }_{\Omega} D_{x}^{-1}$. For this purpose, we write

$$
E_{\Omega}(x)=\sum_{j=0}^{\infty} e_{j}(x)
$$


where functions $e_{j}(x)$ are defined as

$$
e_{j}(x)={ }_{\Omega} D_{x} e_{j+1}(x) \quad \text { or } \quad{ }_{\Omega} D_{x}^{-1} e_{j}(x)=e_{j+1}(x)
$$

with the normalisation $e_{0}(x)=1$. Relation (2.17) now becomes

$$
E_{\Omega}(x)=\sum_{j=0}^{\infty}\left({ }_{\Omega} D_{x}^{-1}\right)^{j} e_{0}(x)=\sum_{j=0}^{\infty}\left({ }_{\Omega} D_{x}^{-1}\right)^{j}
$$

\section{Examples of the Operator $\hat{\Omega}_{\lambda, k}$}

As remarked in Sec. 2, the linear operator $\hat{\Omega}$ is not only multiplicative over functions of $x$, but is also characterised by a deformation parameter $\lambda$. For a complete specification, however, it carries an additional index $k \in \mathbb{Z}^{+}$signifying the degree of the operator $\hat{\Omega}$. We illustrate this by considering a class of operators given by

$$
\hat{\Omega}_{\lambda, k}=\exp \left(-\lambda x^{k+1} \partial_{x}\right)
$$

and study their action on functions of the real variable $x$.

Proposition 3 If $f(x)=x^{n},\left(n \in \mathbb{Z}^{+}\right)$, then

$$
\hat{\Omega}_{\lambda, k} x^{n}=\left\{\frac{x}{\left(1+\lambda k x^{k}\right)^{\frac{1}{k}}}\right\}^{n}
$$

Proof: Let us consider the operation

$$
\begin{aligned}
\exp \left(-\lambda x^{k+1} \partial_{x}\right) x^{n} & =\sum_{n=0}^{\infty} \frac{(-\lambda)^{n}}{n !}\left(x^{k+1} \partial_{x}\right)^{n} x^{n} \\
& =x^{n}+(-\lambda) n x^{k+n}+\frac{(-\lambda)^{2}}{2 !} n(n+k) x^{2 k+n}+\cdots \\
& =x^{n}\left\{1+\left(\frac{-n}{k}\right)\left(\lambda k x^{k}\right)+\frac{1}{2 !}\left(\frac{-n}{k}\right)\left(\frac{-n}{k}-1\right)\left(\lambda k x^{k}\right)^{2}+\cdots\right\} \\
& =x^{n}\left(1+\lambda k x^{k}\right)^{-\frac{n}{k}} \\
& =\left\{\frac{x}{\left(1+\lambda k x^{k}\right)^{\frac{1}{k}}}\right\}^{n}
\end{aligned}
$$

On the other hand, if we consider another class of operators

$$
\hat{\Omega}_{\lambda, k}=\exp \left(-\frac{\lambda}{2}\left\{\hat{x}^{k+1}, \partial_{x}\right\}\right)
$$

where $\{$,$\} is the anticommutator, then their operation given by the adjoint action on the$ position operator $\hat{x}$ results in the equivalence of (3.1) and (3.4) which can be readily seen as follows:

$$
\begin{aligned}
\hat{\Omega}_{\lambda, k} \hat{x} \hat{\Omega}_{\lambda, k}^{-1}= & \exp \left(-\frac{\lambda}{2}\left\{\hat{x}^{k+1}, \partial_{x}\right\}\right) \hat{x} \exp \left(\frac{\lambda}{2}\left\{\hat{x}^{k+1}, \partial_{x}\right\}\right) \\
=\hat{x}+ & {\left[-\frac{\lambda}{2}\left\{\hat{x}^{k+1}, \partial_{x}\right\}, \hat{x}\right] } \\
& +\frac{1}{2 !}\left[-\frac{\lambda}{2}\left\{\hat{x}^{k+1}, \partial_{x}\right\},\left[-\frac{\lambda}{2}\left\{\hat{x}^{k+1}, \partial_{x}\right\}, \hat{x}\right]\right]+\cdots
\end{aligned}
$$


using the Baker-Campbell-Hausdorff formula. The evaluation of the nested commutators proceeds through the following steps

$$
\begin{aligned}
{\left[-\frac{\lambda}{2}\left\{\hat{x}^{k+1}, \partial_{x}\right\}, \hat{x}\right] } & =-\frac{\lambda}{2}\left[\hat{x}^{k+1} \partial_{x}+\partial_{x} \hat{x}^{k+1}, \hat{x}\right] \\
& =-\lambda \hat{x}^{k+1} \\
& =\hat{x}\left(-\frac{1}{k}\right)\left(\lambda k \hat{x}^{k}\right) \\
{\left[-\frac{\lambda}{2}\left\{\hat{x}^{k+1}, \partial_{x}\right\},\left[-\frac{\lambda}{2}\left\{\hat{x}^{k+1}, \partial_{x}\right\}, \hat{x}\right]\right] } & =\left[-\frac{\lambda}{2}\left\{\hat{x}^{k+1}, \partial_{x}\right\},-\lambda \hat{x}^{k+1}\right] \\
& =\lambda^{2}(k+1) \hat{x}^{2 k+1} \\
& =\hat{x}\left(-\frac{1}{k}\right)\left(-\frac{1}{k}-1\right)\left(\lambda k \hat{x}^{k}\right)^{2}
\end{aligned}
$$

and so on. All these terms add up to give

$$
\begin{aligned}
\hat{\Omega}_{\lambda, k} \hat{x} \hat{\Omega}_{\lambda, k}^{-1} & =\hat{x}\left\{I+\left(-\frac{1}{k}\right)\left(\lambda k \hat{x}^{k}\right)+\frac{1}{2 !}\left(-\frac{1}{k}\right)\left(-\frac{1}{k}-1\right)\left(\lambda k \hat{x}^{k}\right)^{2}+\cdots\right\} \\
& =\frac{\hat{x}}{\left(I+\lambda k \hat{x}^{k}\right)^{\frac{1}{k}}}
\end{aligned}
$$

leading finally to the result

$$
\hat{\Omega}_{\lambda, k} \hat{x}^{n} \hat{\Omega}_{\lambda, k}^{-1}=\left\{\frac{\hat{x}}{\left(1+\lambda k \hat{x}^{k}\right)^{\frac{1}{k}}}\right\}^{n}
$$

which is precisely the same as (3.2). We, therefore, conclude that the class of operators (3.1) and (3.4) are essentially equivalent.

A cursory inspection of the operators $\hat{\Omega}_{\lambda, k}$ (邽.1) or (3.4) $)$ helps in defining the relations

$$
\left(\hat{\Omega}_{\lambda, k}\right)^{-1}=\hat{\Omega}_{-\lambda, k}
$$

and

$$
\hat{\Omega}_{\lambda, k} \circ \hat{\Omega}_{\mu, k}=\hat{\Omega}_{\lambda+\mu, k}
$$

These exhibit interesting consequences in that if $\hat{\Omega}_{\lambda, k} x=y$, then $\hat{\Omega}_{\lambda, k}^{-1} y=x$. Writing explicitly, if we have

$$
y=\hat{\Omega}_{\lambda, k} x=\frac{x}{\left(1+\lambda k x^{k}\right)^{\frac{1}{k}}}
$$

then

$$
x=\hat{\Omega}_{\lambda, k}^{-1} y=\frac{y}{\left(1-\lambda k y^{k}\right)^{\frac{1}{k}}}
$$

where we have made use of (3.3) for the case when $n=1$. This is an interesting off-shoot of the foregoing considerations, allowing construction of a class of other operators possessing this unique property.

In more general terms, let us consider the operator

$$
\hat{\Omega}_{\lambda, \mu}=\exp \left(\lambda f(x) \partial_{x}\right)
$$

such that $\hat{\Omega}_{\lambda, \mu} x=y$. In view of the above structures, the functions $f(x)$ and $f(y)$ are invertible and therefore admit the forms

$$
f(y)=\frac{f(x)}{1+\lambda f(x)}, \quad f(x)=\frac{f(y)}{1-\lambda f(y)}
$$


For instance, if we compute the action of $\hat{\Omega}_{\lambda, \mu}$ on $f(x)=\exp (-\mu x)$, say, we find

$$
\hat{\Omega}_{\lambda, \mu} x=x+\mu^{-1} \ln \left[1+\lambda \mu e^{-\mu x}\right]
$$

which constitutes a two-parameter $(\lambda, \mu)$ deformation of the derivative operator. Note, however, that $\hat{\Omega}_{\lambda, \mu} \rightarrow I$ in the limiting case when $\lambda \rightarrow 0$ which is in complete conformity with what we expect. In a similar way, we also evaluate the limit of $\hat{\Omega}_{\lambda, \mu} x$ (3.15) when $\mu \rightarrow 0$ with the result

$$
\lim _{\mu \rightarrow 0}\left\{x+\mu^{-1}\left(\lambda \mu e^{-\mu x}\right)+O(\mu)\right\}=x+\lambda
$$

This is obviously a manifestation of the finite difference operator $\lim _{\mu \rightarrow 0} \hat{\Omega}_{\lambda, \mu}=\exp \left(\lambda \partial_{x}\right)$, characterised by the parameter $\lambda$. We remark in passing that the form (3.15) can be alternatively represented as

$$
\hat{\Omega}_{\lambda, \mu} x=\mu^{-1} \ln \left[e^{\mu x}+\lambda \mu\right]
$$

\subsection{The associated derivative}

The operation of the deformed derivative associated with $\hat{\Omega}_{\lambda, k}$ on a function $f(x)$ can be expressed as

$$
\Omega_{\lambda, k} D_{x} f(x)=\frac{f\left(\hat{\Omega}_{\lambda, k} x\right)-f(x)}{\left(\hat{\Omega}_{\lambda, k} x\right)-x}
$$

Now

$$
\begin{aligned}
\left(\hat{\Omega}_{\lambda, k} x\right)-x & =\frac{x}{\left(1+\lambda k x^{k}\right)^{\frac{1}{k}}}-x \\
& =x\left\{\frac{1-\left(1+\lambda k x^{k}\right)^{\frac{1}{k}}}{\left(1+\lambda k x^{k}\right)^{\frac{1}{k}}}\right\}
\end{aligned}
$$

so that

$$
\Omega_{\lambda, k} D_{x} f(x)=\frac{\left(1+\lambda k x^{k}\right)^{\frac{1}{k}}}{x\left[1-\left(1+\lambda k x^{k}\right)^{\frac{1}{k}}\right]}\left\{f\left(\frac{x}{\left(1+\lambda k x^{k}\right)^{\frac{1}{k}}}\right)-f(x)\right\}
$$

If we choose $f(x)=x^{n}$, then

$$
\Omega_{\lambda, k} D_{x} x^{n}=[[n]]_{\lambda, k} x^{n-1}
$$

where

$$
\begin{aligned}
{[[n]]_{\lambda, k}=[[n]]_{\lambda, k}(x) } & =\frac{\left\{\frac{1}{\left(1+\lambda k x^{k}\right)^{\frac{1}{k}}}\right\}^{n}-1}{\left\{\frac{1}{\left(1+\lambda k x^{k}\right)^{\frac{1}{k}}}\right\}-1} \\
& =\sum_{n=0}^{\infty}\left(1+\lambda k x^{k}\right)^{-\frac{n}{k}}
\end{aligned}
$$

Note that $[[n]]_{\lambda, k}$ is now a function of $x$.

\subsection{Deformed inverse eigenfunctions}

We have already seen that the $\Omega$-exponential functions $E_{\lambda}(x)$ are the eigenfunctions of ${ }_{\Omega} D_{x}$. This enables us to write down the eigenvalue equation

$$
{ }_{\Omega} D_{x} E_{\lambda}(\mu x)=\mu E_{\lambda}(\mu x)
$$


where $\mu$ is regarded as an eigenvalue of ${ }_{\Omega} D_{x}$ with the corresponding eigenfunction $E_{\lambda}(\mu x)$. Explicitly, this can be written as

$$
\hat{\Omega}_{\lambda, k} E_{\lambda}(\mu x)=\left\{1+\mu\left(\hat{\Omega}_{\lambda, k}-I\right) x\right\} E_{\lambda}(\mu x)
$$

which reduces to

$$
\hat{\Omega}_{\lambda, k} E_{\lambda}(x)=\left\{1+\left(\hat{\Omega}_{\lambda, k}-1\right) x\right\} E_{\lambda}(x)
$$

for $\mu=1$. Rewriting (3.25), we have

$$
\begin{aligned}
E_{\lambda}(\mu x) & =\hat{\Omega}_{\lambda, k}^{-1}\left\{1+\mu\left(\hat{\Omega}_{\lambda, k}-1\right) x\right\} E_{\lambda}(\mu x) \\
& =\left\{1+\mu\left(1-\hat{\Omega}_{\lambda, k}^{-1}\right) x\right\} E_{\lambda}\left(\mu \hat{\Omega}_{\lambda, k}^{-1} x\right) \\
& =\left\{1+\mu\left(1-\hat{\Omega}_{-\lambda, k}\right) x\right\} E_{\lambda}\left(\mu \hat{\Omega}_{-\lambda, k} x\right)
\end{aligned}
$$

where use has been made of the relation $\hat{\Omega}_{\lambda, k}^{-1}=\hat{\Omega}_{-\lambda, k}$. Letting $\lambda \rightarrow-\lambda$ and $\mu=-1$ in (3.29), we obtain

$$
\begin{aligned}
E_{-\lambda}(-x) & =\left\{1+\left(\hat{\Omega}_{\lambda, k}-1\right) x\right\} E_{-\lambda}\left(-\hat{\Omega}_{\lambda, k} x\right) \\
& =\left\{1+\left(\hat{\Omega}_{\lambda, k}-1\right) x\right\} \hat{\Omega}_{\lambda, k} E_{-\lambda}(-x)
\end{aligned}
$$

Equations (3.26) and (3.31) lead to

$$
\hat{\Omega}_{\lambda, k} E_{\lambda}(x) \hat{\Omega}_{\lambda, k} E_{-\lambda}(-x)=E_{\lambda}(x) E_{-\lambda}(-x)
$$

i.e.

$$
\hat{\Omega}_{\lambda, k}\left\{E_{\lambda}(x) E_{-\lambda}(-x)\right\}=E_{\lambda}(x) E_{-\lambda}(-x)
$$

or

$$
\left(\hat{\Omega}_{\lambda, k}-I\right)\left\{E_{\lambda}(x) E_{-\lambda}(-x)\right\}=0
$$

Hence

$$
\Omega_{\lambda, k} D_{x}\left\{E_{\lambda}(x) E_{-\lambda}(-x)\right\}=0
$$

which clearly shows that the product $E_{\lambda}(x) E_{-\lambda}(-x)$ under the action of the deformed derivative operator $\Omega_{\lambda, k} D_{x}$ differentiates to zero. Ordinarily, we would expect that only constant functions are endowed with this property. An important consequence of this observation is that any function $F(x)$, not necessarily a constant, with the property $F(x)=F(\hat{\Omega} x)$ will differentiate to zero under the action of the generalised derivative $\Omega_{\lambda, k} D_{x}$.

We now consider the case where $\hat{\Omega}_{\lambda, k}$ is identified with the translation operator, i.e., $\hat{\Omega}_{\lambda, k}=$ $\hat{H}=e^{h \partial_{x}}$. It is then easy to see from (3.26) that

$$
\begin{aligned}
\hat{H} E_{h}(x) & =\{1+(\hat{H}-1) x\} E_{h}(x) \\
& =(1+h) E_{h}(x)
\end{aligned}
$$

Consequently, the eigenfunctions of the translation operator $\hat{H}$ are exactly the same as those of the deformed derivative operator associated with $\hat{H}$. On the other hand, if $\hat{\Omega}_{\lambda, k}$ is taken to be the $q$-dilation operator, i.e., $\hat{\Omega}_{\lambda, k}=\hat{Q}=\exp \left(\ln (q) x \partial_{x}\right)$, then we obtain

$$
E_{q}^{-1}(x)=E_{q^{-1}}(-x)
$$

as expected. 


\section{The Conformal (Möbius) Transformation}

The mathematical framework developed thus far will now be cast in a proper perspective so as to make contact with situations that hold promise for possible applications. The translation operator $\hat{H}$ and the $q$-dilation operator $\hat{Q}$, which give rise to the finite difference derivative and the $q$-derivative, respectively, may be regarded as generators of a one-dimensional affine group. In view of our consideration of the deformed derivative operator, of which $\hat{H}$ and $\hat{Q}$ are special cases, it would seem instructive to visualise a situation where the operator $\hat{\Omega}_{\lambda, k}$, affecting the generalisation, can indeed be assigned the role of an additional generator, resulting in some larger algebraic structure. To this end, we take recourse to the special conformal (Möbius) transformation and carry out an embedding in conjunction with the generators $\hat{H}$ and $\hat{Q}$.

The action of a matrix $M=\left(\begin{array}{ll}a & b \\ c & d\end{array}\right)$ on a variable $x$ is defined as

$$
M \triangleright x=\frac{a x+b}{c x+d}, \quad a d \neq b c
$$

The operator $\hat{\Omega}_{\lambda, k}$ (cf. (3.1) ) for $k=1$ takes the form

$$
\hat{\Omega}=\hat{\Omega}_{\lambda, k=1}=\exp \left(-\lambda x^{2} \partial_{x}\right)
$$

whose action on a variable $x$ (cf. (3.2) ) is given by

$$
\hat{\Omega} x=\frac{x}{1+\lambda x}
$$

Defining the $\Omega, Q$ and $H$ matrices as

$$
\Omega=\left(\begin{array}{cc}
1 & 0 \\
\lambda & 1
\end{array}\right), \quad Q=\left(\begin{array}{cc}
q^{\frac{1}{2}} & 0 \\
0 & q^{-\frac{1}{2}}
\end{array}\right), \quad H=\left(\begin{array}{cc}
1 & h \\
0 & 1
\end{array}\right)
$$

the corresponding actions on $x$ are represented as

$$
\Omega \triangleright x=\frac{x}{\lambda x+1}, \quad Q \triangleright x=q x, \quad H \triangleright x=x+h
$$

The general Möbius operator $M$ is then obtained via the composition

$$
\begin{aligned}
M & =\Omega Q H \\
\text { i.e. } \quad\left(\begin{array}{ll}
a & b \\
c & d
\end{array}\right) & =\left(\begin{array}{cc}
q^{\frac{1}{2}} & q^{\frac{1}{2}} h \\
q^{\frac{1}{2}} \lambda & q^{\frac{1}{2}} \lambda h+q^{-\frac{1}{2}}
\end{array}\right)
\end{aligned}
$$

Equivalently, we express the Möbius transformations on the variable $x$ in the form

$$
\begin{aligned}
\hat{M} x & =\hat{\Omega} \hat{Q} \hat{H} x \\
& =\exp \left[-\lambda x^{2} \partial_{x}+\ln (q) x \partial_{x}+h \partial_{x}\right] x
\end{aligned}
$$

It turns out that $a d-b c=1$. Thus the set of $2 \times 2$ matrices $M$ generate the $S L(2)$ group structure. In other words, the operator $\hat{\Omega}$ serves as an extra generator, in addition to the other two generators typified by the translation operator $\hat{H}$ and the $q$-dilation operator $\hat{Q}$, to generate the group $S L(2)$ as well as its subgroups. The operator $\hat{\Omega}$ is designated as the reduced Möbius transformation operator and conforms to the realisation of the differential operators in view of the transformation (4.1). 


\section{Concluding Remarks}

A modest attempt has been made to construct a generalised version of the deformed derivative operators within the context of a linear operator $\hat{\Omega}$ whose action on functions of a real variable is considered to be multiplicative. The veracity of the mathematical formulation is checked against suitable illustrative examples. We note that the familiar forms of the finite difference and the $q$-derivatives are readily obtained as particular cases of the generalised structure. Such an observation lends credence to the validity of the procedure adopted in carrying out this construction. We have also addressed, though briefly, the possibility of casting the operator $\hat{\Omega}$ in a format compatible with the special conformal or reduced Möbius transformation operator. The inclusion of this operator, along with the translation operator $\hat{H}$ and the $q$-dilation operator $\hat{Q}$, to generate the $S L(2)$ group is expected to be significant in seeking further applications to situations of physical interest such as the conformal transformations. The construction also forms a natural paradigm to investigate generalisation of the $q$-oscillators and the $q$-calculus.

\section{Acknowledgements}

The first author is grateful to Prof. E. Zeidler for the kind hospitality at the Max Planck Institute for Mathematics in the Sciences, Leipzig, where this work was carried out. The second author is supported by a research fellowship of the Royal Commission for the Exhibition of 1851 .

\section{References}

[1] F. H. Jackson, On $q$-functions and a certain difference operator, Trans. Royal Soc. Edinburgh 46 (1908) 253-281; Basic Integration, Quart. J. Math. Oxford Series 2 (1951) 1-16.

[2] M. Arik and D. D. Coon, Hilbert spaces of analytic functions and generalised coherent states, J. Math. Phys. 17 (1976) 524-527.

[3] H. Exton, q-Hypergeometric Functions and Applications, E. Harwood, Chichester (1983).

[4] A. K. Agarwal, E. G. Kalnins and W. Miller, Canonical equations and symmetry techniques for q-series, SIAM J. Math. Anal. 18 (1987) 1519-1538.

[5] V. G. Drinfeld, Quantum groups, In Proc. International Congress of Mathematicians, Academic Press, New York (1987) pp 798-820.

[6] L. D. Faddeev, N. Y. Reshetikhin and L. A. Takhtajan, Quantisation of Lie groups and Lie algebras, Leningrad Math. J. 1 (1990) 193-225.

[7] M. Arik, The $q$-difference operator, the quantum hyperplane, Hilbert spaces of analytic functions and $q-$-oscillators, Z. Phys. C 51 (1991) 627-632.

[8] L. C. Biedenharn and M. A. Lohe, Quantum Group Symmetry and q-Tensor Algebras, World Scientific, Singapore (1995).

[9] A. Klimyk and K. Schmüdgen, Quantum Groups and Their Representations, Springer-Verlag (1997), and references therein. 